\title{
Centralizers of the infinite symmetric group
}

\author{
Zajj Daugherty|" and Peter Herbrich $\|^{k}$
}

Department of Mathematics, Dartmouth College, USA

\begin{abstract}
We review and introduce several approaches to the study of centralizer algebras of the infinite symmetric group $S_{\infty}$. Our work is led by the double commutant relationship between finite symmetric groups and partition algebras; in the case of $S_{\infty}$, we obtain centralizer algebras that are contained in partition algebras. In view of the theory of symmetric functions in non-commuting variables, we consider representations of $S_{\infty}$ that are faithful and that contain invariant elements; namely, non-unitary representations on sequence spaces.

Résumé. Nous étudions les algèbres du centralisateur du groupe symétrique infini $S_{\infty}$, passant en revue certaines approches et en introduisant de nouvelles. Notre travail est basé sur la relation du double commutant entre le groupe symétrique fini et les algèbres de partition; dans le cas de $S_{\infty}$, nous obtenons des algèbres du centralisateur contenues dans les algèbres de partition. Compte tenu de la théorie des fonctions symétriques en variables non commutatives, nous considérons les représentations de $S_{\infty}$ qui sont fidèles et contiennent les invariants; c'est-à-dire, les représentations non unitaires sur les espaces de suites.
\end{abstract}

Keywords: representation theory; infinite symmetric group; centralizer algebras; partition algebras; symmetric functions; Banach spaces

\section{Introduction}

Classical Schur-Weyl duality relates the representation theory of the general linear group $\mathrm{GL}_{n}(\mathbb{C})$ and the finite symmetric group $S_{k}$ via their commuting actions on a common vector space. Namely, if $V \cong$ $\mathbb{C}^{n}$, then the diagonal action of $\mathrm{GL}(V) \cong \mathrm{GL}_{n}(\mathbb{C})$ on the algebraic $k$-fold tensor product $V^{\otimes k}$ fully centralizes the permutation action of $S_{k}$ on the tensor factors:

$$
\mathbb{C} S_{k} \cong \operatorname{End}_{\mathrm{GL}(V)}\left(V^{\otimes k}\right) \quad \text { when } n=\operatorname{dim}(V) \geq k .
$$

In [VT07], Tsilevich and Vershik extend this setting to study the infinite symmetric group $S_{\infty}$ by creating an infinite tensor power $V^{\otimes \infty}$ on which $\mathrm{GL}(V)$ and $S_{\infty}$ share commuting actions. In contrast, we let $V$ have infinite dimension which allows for faithful actions of $S_{\infty}$ on its finite tensor powers.

The group $\mathrm{GL}_{n}(\mathbb{C})$ naturally contains $S_{n}$ as the set of $n \times n$ permutation matrices; in particular, any $V \cong \mathbb{C}^{n}$ can be viewed as an $S_{n}$-module. The centralizer of the corresponding diagonal action of $S_{n}$ on $V^{\otimes k}$ is called the partition algebra $P_{k}(n)$. The partition algebras arose independently in the studies

\footnotetext{
*Email: zajj.b.daugherty@dartmouth.edu. Supported by NSF grant DMS-1162010.

${ }^{\dagger}$ Email: peter.herbrichedartmouth.edu.
} 
of Martin [Mar91, Mar94, Mar96, Mar00] and Jones [Jon94] as generalizations of the Temperley-Lieb algebras and the Potts model in statistical mechanics, respectively. Their work coalesces the study of several tensor power centralizer algebras, including the group algebras of finite symmetric groups, the Temperley-Lieb algebras, the Brauer algebras, and the algebras of uniform block permutations; all of these are examples of diagram algebras, which we discuss in Section 2 .

In [SS13], Sam and Snowden provide a first approach for treating $\mathbb{C} S_{\infty}$ as a finite tensor power centralizer algebra. Their work arrives amongst a recently rejuvenated effort to understand representationtheoretic stability of chains of groups, most salient being the chain of finite symmetric groups $S_{1} \hookrightarrow$ $S_{2} \hookrightarrow S_{3} \hookrightarrow \cdots$. For instance, Bowman, De Visscher, and Orellana [BdVO] use the representationtheoretic duality between $S_{n}$ and $P_{k}(n)$ to study stability in decomposition numbers of representations of symmetric groups. Church, Ellenberg, and Farb [CEF12] use category-theoretic methods to create corresponding chains of modules, all of whose structures are tied together into a single FI-module. Sam and Snowden's approach is related, but they additionally make the connection to Schur-Weyl duality, and treat other examples of groups. Of interest to us is their consideration of the action of $S_{\infty}$ on a countabledimensional vector space $V \cong \mathbb{C}^{(\mathbb{N})}$, as reviewed in Section 3.2. This gives rise to the downwards and upwards partition categories, whose homogeneous degree $k$ components form subalgebras of partition algebras that we call the bottom-propagating and top-propagating partition algebras, respectively.

Another motivation for the study of $S_{\infty}$ originates from the connections between Hopf algebras and symmetric functions. An influential result of Gessel [Ges84] says that the Hopf algebra structure of the Solomon descent algebra is in duality with the algebraic structure of the quasi-symmetric functions, and vice versa. In [MR95], Malvenuto and Reutenauer revisit this connection by essentially using the classical relationship between $\mathrm{GL}_{n}(\mathbb{C})$ and $S_{k}$ as given in $(1 \mathbb{1}$; they exploit the Hopf algebra structure of the tensor algebra

$$
T(V)=\bigoplus_{k=0}^{\infty} V^{\otimes k}, \quad \text { where } V \cong \mathbb{C}^{n}
$$

as well as its bi-module structure for $\operatorname{GL}(V)$ and $\bigoplus_{k \geq 0} \mathbb{C} S_{k}$, which restricts to the work of Gessel. In [AO08], Aguiar and Orellana generalize [MR95] by drawing on the centralizer relationship between the complex reflection groups $C_{r} 2 S_{n}$, which assume the role of $\mathrm{GL}_{n}(\mathbb{C})$, and the subalgebra $U_{k}$ of the partition algebra spanned by uniform block permutations, introduced in [Tan97]. Their result is a graded Hopf algebra $U=\bigoplus_{k>0} U_{k}$, which also contains the Hopf algebra NCSym of symmetric functions in non-commuting variables. However, there is a subtlety in the centralizer relationship between $C_{r} 2 S_{n}$ and $U_{k}$ necessitating $r$ and $n$ to be kept large relative to $k$. This suggests that a study of the entire Hopf algebra $U$ at once might involve $S_{\infty}$.

The above-mentioned approach of Sam and Snowden [SS13] cannot facilitate this role for $S_{\infty}$ since it is missing the $S_{\infty}$-invariant structure crucial for applications to symmetric functions. Namely, consider first the finite-dimensional case, where $V$ has basis $\left\{v_{1}, \ldots, v_{n}\right\}$ over $\mathbb{C}$. Then, $V^{\otimes k}$ can be canonically identified with the space of homogeneous polynomials of degree $k$ in non-commuting variables $v_{1}, \ldots, v_{n}$; the tensor algebra in (2) is isomorphic to the full polynomial ring. The symmetric functions NCSym correspond to the elements of this algebra that are invariant under the permutation action of $S_{n}$ on $v_{1}, \ldots, v_{n}$; in each homogeneous degree $k$, symmetric functions correspond to the invariant elements of $V^{\otimes k}$. For 
example,

$$
(V)^{S_{n}}=\mathbb{C}\left\{\sum_{i=1}^{n} v_{i}\right\} \quad \text { and } \quad(V \otimes V)^{S_{n}}=\mathbb{C}\left\{\sum_{i=1}^{n} v_{i} \otimes v_{i}, \sum_{i, j=1}^{n} v_{i} \otimes v_{j}\right\} .
$$

In order to treat all symmetric functions at once, one passes to the case of countably many non-commuting variables $\left\{v_{i}\right\}_{i \in \mathbb{N}}=\left\{v_{1}, v_{2}, \ldots\right\}$, on which $S_{\infty}$ acts on subscripts. If $V$ has the countable basis $\left\{v_{i}\right\}_{i \in \mathbb{N}}$ as in [SS13], then there are no non-trivial $S_{\infty}$-invariants in its tensor powers $V^{\otimes k}$ since every element in these vector spaces is a finite linear combination of the basis elements.

In Section 4, we present two choices for the representation space $V$ that feature the desired invariant structure. In each case, $V$ contains a countable linearly independent subset whose image determines the endomorphisms under consideration. In Section 4.1. we equip $\mathbb{N}$ with a weighted counting measure, and let $V$ be the corresponding Banach space of $p$-power summable sequences. The measure is chosen so that $V$ and all of the Banach space completions $\overline{V^{\otimes k}}$ of its finite tensor powers contain the desired $S_{\infty^{-}}$ invariants. Theorem 2 states that the centralizer of the action of $S_{\infty}$ on $\overline{V^{\otimes k}}$ inside the set of bounded endomorphisms is the algebra $U_{k}$ used in [AO08]. In Section 4.2, we let $V$ be the Banach space $\ell^{\infty}$ of bounded sequences, and we use suitable replacements for its algebraic tensor powers and the endomorphisms under consideration. Again, the corresponding faithful representations of $S_{\infty}$ contain the desired invariants, and Theorem 5 states that the corresponding centralizer algebras of $\mathbb{C} S_{\infty}$ are subalgebras of partition algebras, namely, the top-propagating partition algebras.

It is noteworthy that the inclusion of non-trivial $S_{\infty}$-invariants into the representation space comes at a cost; namely, the representations studied in Section 4 are not unitary. The non-unitary representation theory of wild groups, of which $S_{\infty}$ is an example, is largely intractable; we refer to [Oko97] for a survey of the representation theory of $S_{\infty}$. By including the desired $S_{\infty}$-invariants, we acquire representations which are reducible, by design, but not fully decomposable. This is reflected in the fact that the centralizer algebras of the actions of $S_{\infty}$ in Section 4 are small in the sense that the double commutant property present in classical Schur-Weyl duality does not hold here, as discussed in Remark 3

We intend this document as an extended abstract of the full paper of the same title. We therefore omit or sketch proofs of our main results.

Acknowledgements: Author Z. Daugherty would like to thank Aaron Lauve for bringing to her attention the link between diagram Hopf algebras and symmetric functions in non-commuting variables, thus inspiring the question of how to place such functions into a centralizer algebra framework.

\section{Diagram algebras}

A set partition of a set $S$ is a set of pairwise disjoint subsets of $S$, called blocks, whose union is $S$. Fix $k \in \mathbb{N}=\{1,2, \ldots\}$, and denote

$$
[k]=\{1, \ldots, k\} \quad \text { and } \quad\left[k^{\prime}\right]=\left\{1^{\prime}, \ldots, k^{\prime}\right\}
$$

so that $[k] \cup\left[k^{\prime}\right]=\left\{1, \ldots, k, 1^{\prime}, \ldots, k^{\prime}\right\}$ is formally a set with $2 k$ elements. To each set partition of $[k] \cup\left[k^{\prime}\right]$, we associate an equivalence class of graphs, called a $(k$-)diagram, as follows. Consider the set of graphs with vertices $[k] \cup\left[k^{\prime}\right]$, and let two graphs be equivalent if they have the same connected components. To each diagram $d$ associate the set partition of $[k] \cup\left[k^{\prime}\right]$ determined by the connected 
components of any of its representatives. For example,

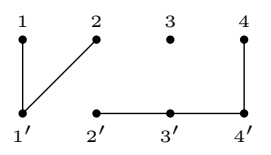

and

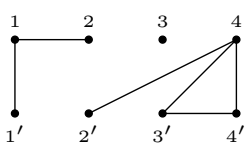

are equivalent, and both represent diagrams for the set partition $\left\{\left\{1,2,1^{\prime}\right\},\{3\},\left\{4,2^{\prime}, 3^{\prime}, 4^{\prime}\right\}\right\}$. Let $D_{k}$ be the set of $k$-diagrams.

The product $d_{1} * d_{2}$ of two diagrams $d_{1}$ and $d_{2}$ is defined as the concatenation of $d_{1}$ above $d_{2}$, where one identifies the bottom vertices of $d_{1}$ with the top vertices of $d_{2}$, and removes any components consisting only of middle vertices. This defines the partition monoid, which can be extended to an algebra as follows. Let $\mathbb{C}(x)$ be the field of rational functions with complex coefficients in an indeterminate $x$. If there are $m$ middle components in the concatenation of $d_{1}$ and $d_{2}$, let $d_{1} d_{2}=x^{m} d_{1} * d_{2}$, and extend linearly over $\mathbb{C}(x)$. For example,

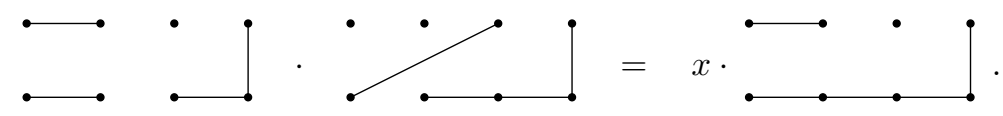

This product is associative and independent of the graphs chosen to represent the partition diagrams.

The partition algebra $P_{k}(x)$ is the span over $\mathbb{C}(x)$ of the set $D_{k}$ of $k$-diagrams equipped with this product, where $P_{0}(x)=\mathbb{C}(x)$. It is an associative algebra with identity given by the diagram corresponding to $\left\{\left\{1,1^{\prime}\right\}, \ldots,\left\{k, k^{\prime}\right\}\right\}$. The dimension of $P_{k}(x)$ is the number of set partitions of $2 k$ elements, that is, the Bell number $B(2 k)$.

Each partition algebra contains many important subalgebras, including group algebras of finite symmetric groups, Brauer algebras, and Temperley-Lieb algebras; see [HR05, Section 1]. In later sections, we will encounter the subalgebras $U_{k}, T P_{k}$, and $B P_{k}$ of $P_{k}(x)$ that share the property that concatenating diagrams in each of these algebras does not lead to middle components; in particular, these algebras can be defined over $\mathbb{C}$. The algebra $U_{k}$ of uniform block permutations is spanned by the set of diagrams $d \in D_{k}$ satisfying

$$
|B \cap[k]|=\left|B \cap\left[k^{\prime}\right]\right| \quad \text { for every block } B \in d .
$$

For example,

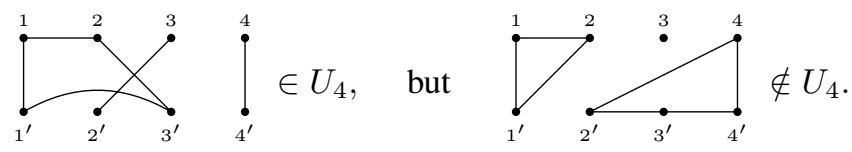

The algebra $U_{k}$ appears in Section 4.1, we refer to Remark 4 in particular. In the following, we say that a block of a diagram is propagating if it contains vertices in both the top and bottom row of the diagram. The top-propagating partition algebra $T P_{k}$ is spanned by the set of diagrams in $D_{k}$ all of whose blocks that contain top vertices are propagating, that is,

$$
T P_{k}=\mathbb{C}\left\{d \in D_{k} \mid \text { for every block } B \in d: B \cap[k] \neq B\right\} .
$$


Similarly, the bottom-propagating partition algebra $B P_{k}$ is spanned by the set of diagrams in $D_{k}$ with no blocks isolated to the bottom row. For example,

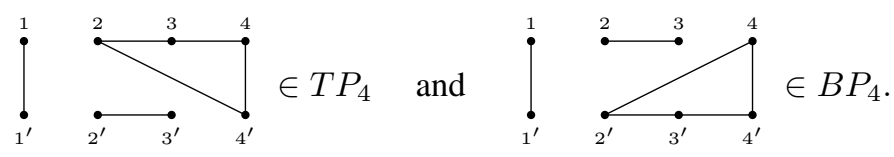

The algebras $T P_{k}$ and $B P_{k}$ appear in Sections 3.2 and 4.2 , respectively.

\section{Vector spaces of finite or countable dimension as permutation modules}

\subsection{Finite symmetric group $S_{n}$ and its action on $\left(\mathbb{C}^{n}\right)^{\otimes k}$}

Let $V$ denote the $n$-dimensional permutation representation of the symmetric group $S_{n}$. That is, let $V$ have basis $\left\{v_{1}, \ldots, v_{n}\right\}$, on which $S_{n}$ acts by $\sigma \cdot v_{i}=v_{\sigma(i)}$ for $\sigma \in S_{n}$. For $\mathbf{i}=\left(i_{1}, \ldots, i_{k}\right) \in[n]^{k}$, a $k$-tuple of integers in $\{1, \ldots, n\}$, and $\sigma \in S_{n}$, we let

$$
v_{\mathbf{i}}=v_{i_{1}} \otimes \cdots \otimes v_{i_{k}} \in V^{\otimes k} \quad \text { and } \quad \sigma(\mathbf{i})=\left(\sigma\left(i_{1}\right), \ldots, \sigma\left(i_{k}\right)\right) \in[n]^{k} .
$$

Let $S_{n}$ act diagonally on the basis $\left\{v_{\mathbf{i}}\right\}_{\mathbf{i} \in[n]^{k}}$ of $V^{\otimes k}$, that is,

$$
\sigma \cdot v_{\mathbf{i}}=v_{\sigma(\mathbf{i})}
$$

and extend this action linearly to $V^{\otimes k}$. Thus, $V^{\otimes k}$ becomes a module for $S_{n}$.

As in Section 2, arrange the vertices of a $k$-diagram reading $1, \ldots, k$ from left to right in the top row and $1^{\prime}, \ldots, k^{\prime}$ from left to right in the bottom row. For each $k$-diagram $d$ and each pair of $k$-tuples $\left(i_{1}, \ldots, i_{k}\right),\left(i_{1^{\prime}}, \ldots, i_{k^{\prime}}\right) \in[n]^{k}$, we define

$$
d_{\left(i_{1^{\prime}}, \ldots, i_{k^{\prime}}\right)}^{\left(i_{1}, \ldots, i_{k}\right)}= \begin{cases}1 & \text { if } i_{\ell}=i_{m} \text { whenever vertices } \ell \text { and } m \text { are connected in } d \\ 0 & \text { otherwise }\end{cases}
$$

For example,

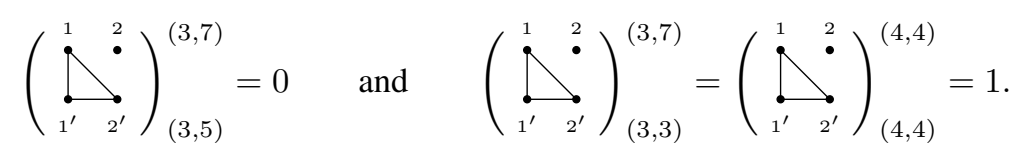

The algebra $P_{k}(n)$ acts on $V^{\otimes k}$; namely, for each $d \in P_{k}(n)$ and $\mathbf{i} \in[n]^{k}$, we define

$$
d \cdot v_{\mathbf{i}}=\sum_{\mathbf{j} \in[n]^{k}} d_{\mathbf{i}}^{\mathbf{j}} v_{\mathbf{j}}
$$

and extend linearly. For example, we have the following identities in the action of $P_{2}(n)$ on $V^{\otimes 2}$

$$
\begin{aligned}
& \square \cdot\left(v_{i} \otimes v_{j}\right)=\delta_{i j} v_{i} \otimes v_{i}, \\
& \because\left(v_{i} \otimes v_{j}\right)=\delta_{i j} \sum_{\ell=1}^{n} v_{\ell} \otimes v_{\ell}, \quad \text { and } \quad \longrightarrow\left(v_{i} \otimes v_{j}\right)=v_{j} \otimes v_{i},
\end{aligned}
$$




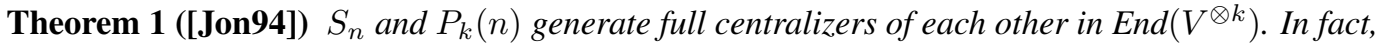

1. $P_{k}(n)$ generates $\operatorname{End}_{S_{n}}\left(V^{\otimes k}\right)$, and when $n \geq 2 k$, we have $P_{k}(n) \cong \operatorname{End}_{S_{n}}\left(V^{\otimes k}\right)$;

2. $S_{n}$ generates $\operatorname{End}_{P_{k}(n)}\left(V^{\otimes k}\right)$.

The proofs of Theorems 2 and 5 are based on the main calculation of Theorem 11, so let us review. Let $A \in \operatorname{End}\left(V^{\otimes k}\right)$ be given by the matrix $\left(A_{\mathbf{i}}^{\mathbf{j}}\right)_{\mathbf{i}, \mathbf{j} \in[n]^{k}}$ such that for each $\mathbf{i} \in[n]^{k}$,

$$
A\left(v_{\mathbf{i}}\right)=\sum_{\mathbf{j} \in[n]^{k}} A_{\mathbf{i}}^{\mathbf{j}} v_{\mathbf{j}}
$$

If $\sigma \in S_{n}$, and $\sigma A=A \sigma$ in $\operatorname{End}\left(V^{\otimes k}\right)$, then for each $\mathbf{i} \in[n]^{k}$,

$$
\sigma A\left(v_{\mathbf{i}}\right)=\sum_{\mathbf{j} \in[n]^{k}} A_{\mathbf{i}}^{\mathbf{j}} v_{\sigma(\mathbf{j})} \quad \text { equals } \quad A \sigma\left(v_{\mathbf{i}}\right)=\sum_{\mathbf{j} \in[n]^{k}} A_{\sigma(\mathbf{i})}^{\mathbf{j}} v_{\mathbf{j}}=\sum_{\mathbf{j} \in[n]^{k}} A_{\sigma(\mathbf{i})}^{\sigma(\mathbf{j})} v_{\sigma(\mathbf{j})},
$$

since $\sigma$ is a bijection of $[n]^{k}$. So for every $\sigma \in S_{n}$, we have

$$
\sigma A=A \sigma \quad \text { if and only if } \quad A_{\sigma(\mathbf{i})}^{\sigma(\mathbf{j})}=A_{\mathbf{i}}^{\mathbf{j}} \quad \text { for every } \mathbf{i}, \mathbf{j} \in[n]^{k} .
$$

Thus, $A \in \operatorname{End}_{S_{n}}\left(V^{\otimes k}\right)$ if and only if the entries of $\left(A_{\mathbf{i}}^{\mathbf{j}}\right)_{\mathbf{i}, \mathbf{j} \in[n]^{k}}$ are uniform on $S_{n}$-orbits, which describes exactly those endomorphisms that come from $P_{k}(n)$.

Note that there are two bases indexed by diagrams commonly arising in the literature. One is the set of matrices with 1's on a single orbit and 0's elsewhere (e.g. the maps $x_{d}$ in [HR05]). The second, given in (4), is a triangular combination of the first, and has the advantage of multiplying by diagram concatenation.

\subsection{Infinite symmetric group $S_{\infty}$ and its action on $\left(\mathbb{C}^{(\mathbb{N})}\right)^{\otimes k}$}

Embed $S_{n} \hookrightarrow S_{n+1}$ as the subgroup which fixes $n+1$. Then, let $S_{\infty}$ be the direct limit of $\left\{S_{n}\right\}_{n \in \mathbb{N}}$, that is, the permutations of $\mathbb{N}$ which fix all but finitely many elements. Let $V$ be a countable-dimensional vector space with basis $\left\{v_{i}\right\}_{i \in \mathbb{N}}$, that is,

$$
V=\mathbb{C}\left\{v_{i}\right\}_{i \in \mathbb{N}}=\left\{\sum_{i \in \mathbb{N}} a_{i} v_{i} \mid a_{i}=0 \text { for all but finitely many } i\right\} \cong \mathbb{C}^{(\mathbb{N})} .
$$

As observed in [SS13], the same calculation as in (5) leads to the same conclusion; namely, $A \in$ $\operatorname{End}_{S_{\infty}}\left(V^{\otimes k}\right)$ if and only if the entries of its matrix representation $\left(A_{\mathbf{i}}^{\mathbf{j}}\right)_{\mathbf{i}, \mathbf{j} \in \mathbb{N}^{k}}$ with respect to $\left\{v_{\mathbf{i}}\right\}_{\mathbf{i} \in \mathbb{N}^{k}}$ are uniform on $S_{\infty}$-orbits. However, since elements of $V^{\otimes k}$ are finite linear combinations of basis elements, we have for every $\mathbf{i} \in \mathbb{N}^{k}$ the set $\left\{\mathbf{j} \in \mathbb{N}^{k} \mid A_{\mathbf{i}}^{\mathbf{j}} \neq 0\right\}$ is finite. Hence, using (4), $\operatorname{End}_{S_{\infty}}\left(V^{\otimes k}\right)$ is spanned by endomorphisms corresponding to top-propagating diagrams, i.e. $\operatorname{End}_{S_{\infty}}\left(V^{\otimes k}\right) \cong T P_{k}$, the top-propagating partition algebra defined in 3 .

In [SS13], Sam and Snowden study the endomorphisms of the tensor algebra

$$
T(V)=\bigoplus_{k=0}^{\infty} V^{\otimes k}
$$


generated by $(k, \ell)$-diagrams with vertices $[k] \cup\left[\ell^{\prime}\right]$, where $k, \ell \in \mathbb{N}$ are not necessarily equal, and with no blocks isolated to the top row. Multiplication between a $\left(k_{1}, \ell_{1}\right)$-diagram and a $\left(k_{2}, \ell_{2}\right)$-diagram is defined as concatenation when $\ell_{1}=k_{2}$, and as zero otherwise. Since no middle components arise in resolving concatenations, this multiplication involves no parameter. It gives rise to the so-called upwards partition algebra $U P$, which is the span over $\mathbb{C}$ of the set of $(k, l)$-diagrams. In contrast to Sam and Snowden [SS13], we write all actions as left actions, for which reason our use of upwards and downwards is opposite to theirs. The top-propagating partition algebra $T P_{k}$ is exactly the homogeneous degree $k$ component of $U P$.

\section{Sequence spaces as permutation modules}

The study of symmetric functions in countably many variables requires to leave the finite-dimensional realm and instead consider vector spaces $V$ that contain countable linearly independent subsets $\left\{v_{i}\right\}_{i \in \mathbb{N}}$. With an eye toward studying $S_{\infty}$-invariants as mentioned in Section 1 , we require $\sum_{i=1}^{\infty} v_{i}$ to be interpretable as an element of $V$. This rules out the possibility of $\left\{v_{i}\right\}_{i \in \mathbb{N}}$ being a Hamel basis of $V$ as in Section 3.2, in which case every vector has a unique expression as a finite linear combination of the basis vectors $\left\{v_{i}\right\}_{i \in \mathbb{N}}$. We propose the following approach:

Choose a vector space $V$ containing a countable linearly independent subset $\left\{v_{i}\right\}_{i \in \mathbb{N}}$ and a vector $\sum_{i=1}^{\infty} v_{i}$ that is invariant under $\mathbb{C} S_{\infty}$, which is considered as a subalgebra of an algebra of endomorphisms of $V$ that are determined by their images on $\left\{v_{i}\right\}_{i \in \mathbb{N}}$.

\section{1 p-power summable sequences}

We recall definitions from Banach space theory that will be used throughout this section. A sequence $\left\{w_{\ell}\right\}_{\ell \in \mathbb{N}}$ in a normed vector space $V$ is called a Cauchy sequence, if for every $\varepsilon>0$ there exists $L \in \mathbb{N}$ such that for all integers $\ell, m>L$, we have $\left\|w_{\ell}-w_{m}\right\|<\varepsilon$. A normed vector space $V$ is called a Banach space if every Cauchy sequence $\left\{w_{\ell}\right\}_{\ell \in \mathbb{N}}$ in $V$ converges to some vector $w=\lim _{\ell \rightarrow \infty} w_{\ell}$ in $V$, meaning $\lim _{\ell \rightarrow \infty}\left\|w-w_{\ell}\right\|=0$. A sequence $\left\{v_{i}\right\}_{i \in \mathbb{N}}$ in a Banach space $V$ is called a Schauder basis if for every $v \in V$ there exist unique scalars $\left\{a_{i}\right\}_{i \in \mathbb{N}}$ such that

$$
v=\sum_{i=1}^{\infty} a_{i} v_{i}=\lim _{\ell \rightarrow \infty} \sum_{i=1}^{\ell} a_{i} v_{i} \quad \text { meaning } \quad \lim _{\ell \rightarrow \infty}\left\|v-\sum_{i=1}^{\ell} a_{i} v_{i}\right\|=0
$$

qualitatively, a Schauder basis is a linearly independent set such that every element of $V$ can be written uniquely as in (6). A Schauder basis $\left\{v_{i}\right\}_{i \in \mathbb{N}}$ is called unconditional if for every $v \in V$ the convergence in (6) is unconditional. If now $A$ is a continuous endomorphism on $V$, and if $v=\sum_{i=1}^{\infty} a_{i} v_{i}$, then $\left\{A\left(v_{i}\right)\right\}_{i \in \mathbb{N}}$ determines $A(v)$ since

$$
A(v)=A\left(\lim _{\ell \rightarrow \infty} \sum_{i=1}^{\ell} a_{i} v_{i}\right)=\lim _{\ell \rightarrow \infty} A\left(\sum_{i=1}^{\ell} a_{i} v_{i}\right)=\lim _{\ell \rightarrow \infty} \sum_{i=1}^{\ell} a_{i} A\left(v_{i}\right) .
$$

We therefore study the following special case of the above-mentioned approach:

Choose a Banach space $V$ with a countable Schauder basis $\left\{v_{i}\right\}_{i \in \mathbb{N}}$ such that $\sum_{i=1}^{\infty} v_{i}$ converges, and study $\mathbb{C} S_{\infty}$ as a subalgebra of the algebra of continuous endomorphisms of $V$. 
To this end, we consider $L^{p}$-spaces of sequences of the form

$$
V=L^{p}\left(\mathbb{N}, \mu^{p}\right)=\left\{v=\left.\left(a_{1}, a_{2}, \ldots\right) \in \mathbb{C}^{\mathbb{N}}\left|\|v\|^{p}=\sum_{i=1}^{\infty}\right| a_{i}\right|^{p} \mu_{i}^{p}<\infty\right\},
$$

where $1 \leq p<\infty$, and $\mu^{p}$ is a weighted counting measure on $\mathbb{N}$, which is determined by a sequence $\left(\mu_{i}\right)_{i \in \mathbb{N}}$ with $\mu_{i}>0$ for all $i \in \mathbb{N}$ via $\mu^{p}(\{i\})=\mu_{i}^{p}$. The space $\ell^{\infty}=L^{\infty}(\mathbb{N}, \mu)$ of bounded sequences will be dealt with in Section 4.2. The normed vector space (7) is a Banach space that has unconditional Schauder bases such as $\left\{v_{i}\right\}_{i \in \mathbb{N}}$ given by $v_{i}=\left(\delta_{i j}\right)_{j \in \mathbb{N}}$, that is, $v_{1}=(1,0,0, \ldots), v_{2}=(0,1,0 \ldots)$, etc.. In particular, $v=\left(a_{1}, a_{2}, \ldots\right) \in V$ if and only if $\sum_{i \in \mathbb{N}} a_{i} v_{i}$ converges unconditionally to $v$ in $V$. This allows to introduce the notation $\sum_{i \in \mathbb{N}} a_{i} v_{i}$ for arbitrary $\left(a_{1}, a_{2}, \ldots\right) \in \mathbb{C}^{\mathbb{N}}$ so that

$$
V=\left\{v=\left.\sum_{i \in \mathbb{N}} a_{i} v_{i} \in \mathbb{C}^{\mathbb{N}}\left|\|v\|^{p}=\sum_{i=1}^{\infty}\right| a_{i}\right|^{p} \mu_{i}^{p}<\infty\right\} .
$$

In order to ensure that $\sum_{i \in \mathbb{N}} v_{i}=(1,1,1, \ldots) \in V$, we henceforth require that $\left(\mu_{i}\right)_{i \in \mathbb{N}} \in \ell^{p}$, that is, $\sum_{i=1}^{\infty} \mu_{i}^{p}<\infty$.

We turn to the algebraic $k$-fold tensor product $V^{\otimes k}=\left(L^{p}\left(\mathbb{N}, \mu^{p}\right)\right)^{\otimes k}$, and note that it carries a canonical cross norm so that its completion $\overline{V^{\otimes k}}$ is isomorphic to $V$ [DF93, Chapter 7]; namely,

$$
\overline{V^{\otimes k}}=L^{p}\left(\mathbb{N}^{k},\left(\mu^{p}\right)^{\times k}\right)=\left\{v=\left.\sum_{\mathbf{i} \in \mathbb{N}^{k}} a_{\mathbf{i}} v_{\mathbf{i}} \in \mathbb{C}^{\mathbb{N}^{k}}\left|\|v\|^{p}=\sum_{\mathbf{i} \in \mathbb{N}^{k}}\right| a_{\mathbf{i}}\right|^{p} \mu_{\mathbf{i}}^{p}<\infty\right\},
$$

where $\sum_{\mathbf{i} \in \mathbb{N}^{k}} a_{\mathbf{i}} v_{\mathbf{i}}$ represents the function $v: \mathbb{N}^{k} \rightarrow \mathbb{C}$ given by $v(\mathbf{i})=a_{\mathbf{i}}$, and for $\mathbf{i}=\left(i_{1}, \ldots, i_{k}\right) \in \mathbb{N}^{k}$,

$$
v_{\mathbf{i}}=v_{i_{1}} \otimes \cdots \otimes v_{i_{k}} \quad \text { and } \quad \mu_{\mathbf{i}}=\prod_{\ell=1}^{k} \mu_{i_{\ell}}=\left\|v_{\mathbf{i}}\right\| .
$$

In particular, $\left\{v_{\mathbf{i}}\right\}_{\mathbf{i} \in \mathbb{N}^{k}}$ is an unconditional Schauder basis of $\overline{V^{\otimes k}}$, and $V^{\otimes k}$ can be identified with the dense subset of linear combinations of vectors of the form

$\sum_{\mathbf{i}=\left(i_{1}, \ldots, i_{k}\right) \in \mathbb{N}^{k}}\left(\prod_{\ell=1}^{k} a_{\ell i_{\ell}}\right) v_{\mathbf{i}}=\left(\sum_{i_{1} \in \mathbb{N}} a_{1 i_{1}} v_{i_{1}}\right) \otimes \ldots \otimes\left(\sum_{i_{k} \in \mathbb{N}} a_{k i_{k}} v_{i_{k}}\right)$ with $\sup _{1 \leq \ell \leq k} \sum_{i_{\ell}=1}^{\infty}\left|a_{\ell i_{\ell}}\right|^{p} \mu_{i_{\ell}}^{p}<\infty$.

A linear operator on a Banach space, say $A: \overline{V^{\otimes k}} \rightarrow \overline{V^{\otimes k}}$, is continuous if and only if it is bounded, meaning it maps bounded sets to bounded sets. This happens precisely if it has finite operator norm,

$$
\|A\|=\sup _{v \in \overline{V^{\otimes k}}:\|v\| \leq 1}\|A(v)\| .
$$

Moreover, the set of bounded operators

$$
\mathcal{B}\left(\overline{V^{\otimes k}}\right)=\left\{A \in \operatorname{End}\left(\overline{V^{\otimes k}}\right) \mid\|A\|<\infty\right\}
$$


is a Banach space with respect to the operator norm.

We let $S_{\infty}$ act diagonally on the basis $\left\{v_{\mathbf{i}}\right\}_{\mathbf{i} \in \mathbb{N}^{k}}$ of $\overline{V^{\otimes k}}$ so that for $\sigma \in S_{\infty}$ and $\mathbf{i}=\left(i_{1}, \ldots, i_{k}\right) \in \mathbb{N}^{k}$,

$$
\sigma \cdot v_{\mathbf{i}}=v_{\sigma(\mathbf{i})}, \quad \text { where } \quad \sigma(\mathbf{i})=\left(\sigma\left(i_{1}\right), \ldots, \sigma\left(i_{k}\right)\right),
$$

and we extend linearly. In other words, if $\sigma \in S_{\infty}$ and $v=\sum_{\mathbf{i} \in \mathbb{N}^{k}} a_{\mathbf{i}} v_{\mathbf{i}} \in \overline{V^{\otimes k}}$, then

$$
\sigma \cdot v=\sum_{\mathbf{i} \in \mathbb{N}^{k}} a_{\mathbf{i}} v_{\sigma(\mathbf{i})}=\sum_{\mathbf{i} \in \mathbb{N}^{k}} a_{\sigma^{-1}(\mathbf{i})} v_{\mathbf{i}} \in \overline{V^{\otimes k}}
$$

Each $\sigma \in S_{\infty}$ gives rise to a continuous endomorphism since it fixes all but finitely many of the vectors $\left\{v_{\mathbf{i}}\right\}_{\mathbf{i} \in \mathbb{N}^{k}}$. Thus, $\mathbb{C} S_{\infty}$ can be regarded as a subalgebra of $\mathcal{B}\left(\overline{V^{\otimes k}}\right)$.

Theorem 2 The centralizer of $\mathbb{C} S_{\infty}$ in $\mathcal{B}\left(\overline{V^{\otimes k}}\right)$ is isomorphic to the finite-dimensional algebra $U_{k}$ of uniform block permutations.

Proof: (A sketch.) Every $A \in \mathcal{B}\left(\overline{V^{\otimes k}}\right)$ is determined by its images on $\left\{v_{\mathbf{i}}\right\}_{\mathbf{i} \in \mathbb{N}^{k}}$, which we arrange in a $\operatorname{matrix}\left(A_{\mathbf{i}}^{\mathbf{j}}\right)_{\mathbf{i}, \mathbf{j} \in \mathbb{N}^{k}} \in \mathbb{C}^{\mathbb{N}^{k} \times \mathbb{N}^{k}}$ such that for each $\mathbf{i} \in \mathbb{N}^{k}$,

$$
A\left(v_{\mathbf{i}}\right)=\sum_{\mathbf{j} \in \mathbb{N}^{k}} A_{\mathbf{i}}^{\mathbf{j}} v_{\mathbf{j}} \in \overline{V^{\otimes k}}
$$

Then using a similar calculation to (5), we determine that $A$ is in the centralizer of $\mathbb{C} S_{\infty}$ if and only if $A$ can be represented as a partition diagram. Then an analysis of which of those operators are bounded (and therefore continuous) operators restricts us to $U_{k}$.

Remark 3 The group algebra $\mathbb{C} S_{\infty}$ does not satisfy the double commutant property in $\mathcal{B}\left(\overline{V^{\otimes k}}\right)$, that is, the centralizer of $U_{k}$ in $\mathcal{B}\left(\overline{V^{\otimes k}}\right)$ strictly contains $\mathbb{C} S_{\infty}$. For example, when $k=1$, the centralizer of $\mathbb{C} S_{\infty}$ in $\mathcal{B}\left(\overline{V^{\otimes 1}}\right)=\mathcal{B}(V)$ is $U_{1}=\mathbb{C}\left\{\mathrm{id}_{V}\right\}$; but the centralizer of $\mathbb{C}\left\{\mathrm{id}_{V}\right\}$ is all of $\mathcal{B}(V)$. This results from the fact that the action of $S_{\infty}$ on $\mathcal{B}(V)$ is not semisimple. In fact, the $S_{\infty}$-invariant subspace $\mathbb{C}\left\{\sum_{i \in \mathbb{N}} v_{i}\right\}$ does not have a closed $\mathbb{C} S_{\infty}$-invariant complement, so that all projection operators with range $\mathbb{C}\left\{\sum_{i \in \mathbb{N}} v_{i}\right\}$ are unbounded. In particular, $V=\overline{V^{\otimes 1}}$ is not fully decomposable as a $\mathbb{C} S_{\infty}-$ module. A similar argument applies for $k>1$.

In view of the connection to symmetric polynomials, we define for each set partition $\pi$ of $[k]$,

$$
\pi_{\left(i_{1}, \ldots, i_{k}\right)}= \begin{cases}1 & \text { if } i_{\ell}=i_{m} \text { whenever } \ell \text { and } m \text { are in the same block of } \pi \\ 0 & \text { otherwise }\end{cases}
$$

and let

$$
m_{\pi}=\sum_{\mathbf{i} \in \mathbb{N}^{k}} \pi_{\mathbf{i}} v_{\mathbf{i}} \in \mathbb{C}^{\mathbb{N}^{k}}
$$

For example,

$$
m_{\{\{1,2,3\}\}}=\sum_{i \in \mathbb{N}} v_{i} \otimes v_{i} \otimes v_{i} \quad \text { and } \quad m_{\{\{1,3\},\{2\}\}}=\sum_{i, j \in \mathbb{N}} v_{i} \otimes v_{j} \otimes v_{i} .
$$


These elements correspond to the so-called monomial symmetric functions of degree $k$ in NCSym, which form a basis for the space of homogeneous symmetric functions of degree $k$ in non-commuting variables. It is straightforward to check that, just as is the case with countable-dimensional vector spaces, the vector space $\left(\overline{V^{\otimes k}}\right)^{S_{\infty}}$ has $\left\{m_{\pi} \mid \pi\right.$ a set partition of $\left.[k]\right\}$ as a basis.

Remark 4 Despite the delicate properties of the action of $S_{\infty}$ on $\overline{V^{\otimes k}}$ mentioned in the previous remark, Theorem 2 frames the vector space $\left(\overline{V^{\otimes k}}\right)^{S_{\infty}}$ of $S_{\infty}$-invariant vectors as a natural module for the algebra $U_{k}$ of uniform block permutations. In fact, in [AO08], Aguiar and Orellana study the combinatorial Hopf algebra of uniform block permutations, and find that the ring of symmetric functions in non-commuting variables naturally lives in their algebra. A priori, this may be surprising since in their setting, $U_{k}$ arises as the centralizer of the seemingly unrelated complex reflection group $C_{r} \prec S_{n}$ on a permutation-like module, as shown in [Tan97]. However, there is a subtlety in the centralizer relationship depending on the values of $k$ and $r$. One can use the action of $C_{r} 2 S_{n}$ on $\mathbb{C}^{n}$ as defined in [AO08. Section 3.1], calculate the corresponding commutation conditions as in (5), and take the limit as $r, n \rightarrow \infty$ to obtain the same commutation conditions as in the proof of Theorem 2 In light of this observation, the results of Aguiar and Orellana connecting the Hopf algebra of uniform block permutations and the ring of symmetric functions in non-commuting variables appear natural.

\subsection{Bounded sequences}

In the following, we consider the Banach space of bounded sequences

$$
\ell^{\infty}=\left\{v=\left(a_{1}, a_{2}, \ldots\right) \in \mathbb{C}^{\mathbb{N}}\left|\|v\|_{\infty}=\sup _{i \in \mathbb{N}}\right| a_{i} \mid<\infty\right\} .
$$

Recall that $\ell^{\infty}$ is not separable, meaning that it has no countable dense subsets. In particular, $\ell^{\infty}$ has no countable Schauder bases. For example, if $\left\{v_{i}\right\}_{i \in \mathbb{N}}$ is given by $v_{i}=\left(\delta_{i j}\right)_{j \in \mathbb{N}}$, then $\left\{\sum_{i=1}^{\ell} v_{i}\right\}_{\ell \in \mathbb{N}}$ is not a Cauchy sequence even though $(1,1,1, \ldots) \in \ell^{\infty}$. Therefore, we no longer use the notation $\sum_{i \in \mathbb{N}} a_{i} v_{i}$ for $\left(a_{1}, a_{2}, \ldots\right) \in \mathbb{C}^{\mathbb{N}}$. Following the previous section, we regard $\left(\ell^{\infty}\right)^{\otimes k}$ as a subspace of

$$
\ell^{\infty}\left(\mathbb{N}^{k}\right)=\left\{v=\left(a_{\mathbf{i}}\right)_{\mathbf{i} \in \mathbb{N}^{k}} \in \mathbb{C}^{\mathbb{N}^{k}}\left|\|v\|_{\infty}=\sup _{\mathbf{i} \in \mathbb{N}^{k}}\right| a_{\mathbf{i}} \mid<\infty\right\}
$$

such that for $\mathbf{i}=\left(i_{1}, \ldots, i_{k}\right) \in \mathbb{N}^{k}$,

$$
v_{\mathbf{i}}=v_{i_{1}} \otimes \cdots \otimes v_{i_{k}}=\left(\delta_{\mathbf{i j}}\right)_{\mathbf{j} \in \mathbb{N}^{k}}, \text { where } \delta_{\mathbf{i j}}= \begin{cases}1 & \text { if } \mathbf{i}=\mathbf{j}, \\ 0 & \text { otherwise }\end{cases}
$$

Note that $\left(\ell^{\infty}\right)^{\otimes k}$ is not dense in $\ell^{\infty}\left(\mathbb{N}^{k}\right)$ if $k>1$. Again, $\left\{v_{\mathbf{i}}\right\}_{\mathbf{i} \in \mathbb{N}^{k}}$ is not a Schauder basis, and bounded operators are not generally determined by their images on $\left\{v_{\mathbf{i}}\right\}_{\mathbf{i} \in \mathbb{N}^{k}}$. Thus, we restrict our consideration to operators $A$ on $\ell^{\infty}\left(\mathbb{N}^{k}\right)$ which are determined by their associated matrix with entries $A_{\mathbf{i}}^{\mathbf{j}}=\left(A\left(v_{\mathbf{i}}\right)\right)_{\mathbf{j}}$ for $\mathbf{i}, \mathbf{j} \in \mathbb{N}^{k}$. To this end, let

$$
\mathcal{B}_{\text {Mat }}\left(\ell^{\infty}\left(\mathbb{N}^{k}\right)\right)=\left\{A=\left(A_{\mathbf{i}}^{\mathbf{j}}\right)_{\mathbf{i}, \mathbf{j} \in \mathbb{N}^{k}} \in \mathbb{C}^{\mathbb{N}^{k} \times \mathbb{N}^{k}} \mid\|A\|_{\text {Mat }}=\sup _{\mathbf{j} \in \mathbb{N}^{k}}\left\{\sum_{\mathbf{i} \in \mathbb{N}^{k}}\left|A_{\mathbf{i}}^{\mathbf{j}}\right|\right\}<\infty\right\}
$$


If $A=\left(A_{\mathbf{i}}^{\mathbf{j}}\right)_{\mathbf{i}, \mathbf{j} \in \mathbb{N}^{k}} \in \mathcal{B}_{\text {Mat }}\left(\ell^{\infty}\left(\mathbb{N}^{k}\right)\right)$ and $v=\left(b_{\mathbf{i}}\right)_{\mathbf{i} \in \mathbb{N}^{k}} \in \ell^{\infty}\left(\mathbb{N}^{k}\right)$, we define for each $\mathbf{j} \in \mathbb{N}^{k}$,

$$
(A(v))_{\mathbf{j}}=\sum_{\mathbf{i} \in \mathbb{N}^{k}} A_{\mathbf{i}}^{\mathbf{j}} b_{\mathbf{i}}
$$

which converges absolutely since

$$
\sum_{\mathbf{i} \in \mathbb{N}^{k}}\left|A_{\mathbf{i}}^{\mathbf{j}}\right|\left|b_{\mathbf{i}}\right| \leq \sum_{\mathbf{i} \in \mathbb{N}^{k}}\left|A_{\mathbf{i}}^{\mathbf{j}}\right|\|v\|_{\infty} \leq\|A\|_{\text {Mat }}\|v\|_{\infty} .
$$

Hence, $A(v) \in \ell^{\infty}\left(\mathbb{N}^{k}\right)$, and $A$ gives rise to an operator on $\ell^{\infty}\left(\mathbb{N}^{k}\right)$ with norm bounded by $\|A\|_{\text {Mat }}$. The norm $\|A\|_{\infty}$ of each $A \in \mathcal{B}_{\text {Mat }}\left(\ell^{\infty}\left(\mathbb{N}^{k}\right)\right)$ as an operator on $\ell^{\infty}\left(\mathbb{N}^{k}\right)$ equals $\|A\|_{\text {Mat }}$, and $\mathcal{B}_{\text {Mat }}\left(\ell^{\infty}\left(\mathbb{N}^{k}\right)\right)$ is a subalgebra of $\mathcal{B}\left(\ell^{\infty}\left(\mathbb{N}^{k}\right)\right)$. Thus this set of operators is a viable space for our study.

We define a norm-preserving action of $S_{\infty}$ on $\ell^{\infty}\left(\mathbb{N}^{k}\right)$ by

$$
\sigma \cdot\left(a_{\mathbf{i}}\right)_{\mathbf{i} \in \mathbb{N}^{k}}=\left(a_{\sigma^{-1}(\mathbf{i})}\right)_{\mathbf{i} \in \mathbb{N}^{k}} \quad \text { for } \sigma \in S_{\infty}
$$

so that for each $\mathbf{i} \in \mathbb{N}^{k}, \sigma \cdot v_{\mathbf{i}}=v_{\sigma(\mathbf{i})}$. As in the $p$-power summable sequences case, the vector space $\left(\ell^{\infty}\left(\mathbb{N}^{k}\right)\right)^{S_{\infty}}$ of $S_{\infty}$-invariant elements in $\ell^{\infty}\left(\mathbb{N}^{k}\right)$ has the functions $\left\{m_{\pi} \mid \pi\right.$ a set partition of $\left.[k]\right\}$ defined by (8) as a basis.

Theorem 5 The centralizer of $\mathbb{C} S_{\infty}$ in $\mathcal{B}_{\text {Mat }}\left(\ell^{\infty}\left(\mathbb{N}^{k}\right)\right)$ is isomorphic to the finite-dimensional bottompropagating partition algebra $B P_{k}$.

Proof: (A sketch.) First show that $A=\left(A_{\mathbf{i}}^{\mathbf{j}}\right)_{\mathbf{i}, \mathbf{j} \in \mathbb{N}^{k}} \in \mathcal{B}_{\text {Mat }}\left(\ell^{\infty}\left(\mathbb{N}^{k}\right)\right)$ is in the centralizer of $\mathbb{C} S_{\infty}$ if and only if it is a linear combination of the finitely many diagram matrices defined by (4). The claim now follows from the observation that if $A=\left(A_{\mathbf{i}}^{\mathbf{j}}\right)_{\mathbf{i}, \mathbf{j} \in \mathbb{N}^{k}}$ is such a linear combination, then $\left\{\mathbf{i} \in \mathbb{N}^{k} \mid A_{\mathbf{i}}^{\mathbf{j}} \neq 0\right\}$ is finite for every $\mathbf{j} \in \mathbb{N}^{k}$ if and only if $A$ is a linear combination of matrices corresponding to diagrams with no blocks isolated to the bottom row, that is, diagrams in $B P_{k}$.

Remark 6 As in Section 4.1 $S_{\infty}$ is strictly contained in its double commutant; see Remark 3 . Similarly to Remark 4 the vector space $\left(\ell^{\infty}\left(\mathbb{N}^{k}\right)\right)^{S_{\infty}}$ becomes a natural module for $B P_{k}$, and it has a basis consisting of elements which may be identified with monomial symmetric functions. Of course, the entire partition algebra $P_{k}(x)$ has a natural action on the set of set partitions of $[k]$ obtained by identifying each set partition $\pi$ with the diagram $d=\pi \cup\left\{\left\{1^{\prime}\right\}, \ldots,\left\{k^{\prime}\right\}\right\}$; these diagrams form a left ideal in $P_{k}(x)$. One might expect an action of $P_{k}(x)$ on the basis $\left\{m_{\pi} \mid \pi\right.$ a set partition of $\left.[k]\right\}$ of $\left(\ell^{\infty}\left(\mathbb{N}^{k}\right)\right)^{S_{\infty}}$. Recall that in Theorem 1] $x$ must be specialized to the number $n$ of basis vectors on which $S_{n}$ acts. The transition $x \rightarrow \infty$ can be carried out rigorously only if middle components are avoided in diagram concatenations, in this case by restricting to $B P_{k}$.

\section{References}

[AO08] Marcelo Aguiar and Rosa C. Orellana, The Hopf algebra of uniform block permutations, J. Algebraic Combin. 28 (2008), no. 1, 115-138.

[BdVO] Christopher Bowman, Maud de Visscher, and Rosa Orellana, The partition algebra and the kronecker coefficients, Trans. Amer. Math. Soc., to appear. 
[CEF12] Thomas Church, Jordan S. Ellenberg, and Benson Farb, Fi-modules: a new approach to stability for $S_{n}$-representations, arXiv:1204.4533v2 (2012).

[DF93] Andreas Defant and Klaus Floret, Tensor norms and operator ideals, North-Holland Mathematics Studies, vol. 176, North-Holland Publishing Co., Amsterdam, 1993.

[Ges84] Ira M. Gessel, Multipartite P-partitions and inner products of skew Schur functions, Combinatorics and algebra (Boulder, Colo., 1983), Contemp. Math., vol. 34, Amer. Math. Soc., Providence, RI, 1984, pp. 289-317.

[HR05] Tom Halverson and Arun Ram, Partition algebras, European J. Combin. 26 (2005), no. 6, 869921.

[Jon94] Vaughan F. R. Jones, The Potts model and the symmetric group, Subfactors (Kyuzeso, 1993), World Sci. Publ., River Edge, NJ, 1994, pp. 259-267.

[Mar91] Paul Martin, Potts models and related problems in statistical mechanics, Series on Advances in Statistical Mechanics, vol. 5, World Scientific Publishing Co. Inc., Teaneck, NJ, 1991.

[Mar94] _ Temperley-Lieb algebras for nonplanar statistical mechanics—-the partition algebra construction, J. Knot Theory Ramifications 3 (1994), no. 1, 51-82.

[Mar96] _ , The structure of the partition algebras, J. Algebra 183 (1996), no. 2, 319-358.

[Mar00] _ The partition algebra and the Potts model transfer matrix spectrum in high dimensions, J. Phys. A 33 (2000), no. 19, 3669-3695.

[MR95] Clauda Malvenuto and Christophe Reutenauer, Duality between quasi-symmetric functions and the Solomon descent algebra, J. Algebra 177 (1995), no. 3, 967-982.

[Oko97] Andrei Okounkov, On representations of the infinite symmetric group, Zap. Nauchn. Sem. S.Peterburg. Otdel. Mat. Inst. Steklov. (POMI) 240 (1997), no. Teor. Predst. Din. Sist. Komb. i Algoritm. Metody. 2, 166-228, 294, English translation in J. Math. Sci. 96 (1999), no. 5, 35503589 .

[SS13] Steven V. Sam and Andrew Snowden, Stability patterns in representation theory, arXiv:1302.5859 (2013).

[Tan97] Kenichiro Tanabe, On the centralizer algebra of the unitary reflection group $G(m, p, n)$, Nagoya Math. J. 148 (1997), 113-126.

[VT07] A. M. Vershik and N. V. Tsilevich, Induced representations of the infinite symmetric group and their spectral theory, Dokl. Akad. Nauk 412 (2007), no. 1, 7-10. 